Daimon. Revista Internacional de Filosofía, n ${ }^{\circ} 85$ (2022), pp. 83-97

ISSN: 1130-0507 (papel) y 1989-4651 (electrónico)

http://dx.doi.org/10.6018/daimon.398251

Licencia Creative Commons Reconocimiento-NoComercial-SinObraDerivada 3.0 España (texto legal). Se pueden copiar, usar, difundir, transmitir y exponer públicamente, siempre que: i) se cite la autoría y la fuente original de su publicación (revista, editorial y URL de la obra); ii) no se usen para fines comerciales; iii) se mencione la existencia y especificaciones de esta licencia de uso.

(c) (1) (9)

\title{
El concepto de muerte como paradoja en la vía del conocimiento absoluto en la filosofía de F. W. J. Schelling
}

\author{
The Concept of Death like Paradox in the Path of Absolute \\ Knowledge in F. W. J. Schelling's Philosophy
}

\author{
MIGUEL ÁNGEL RAMÍREZ CORDÓN*
}

\begin{abstract}
Resumen: Se pretende analizar el concepto de muerte haciendo especial énfasis en las Stuttgarter Privatvorlesungen para establecer que el filósofo emplea este concepto con diversas acepciones, lo cual le lleva a englobar el ámbito de la esencia y de lo ente como ámbitos de muerte siempre que son considerados aisladamente. Ha de haber entre estos dos parámetros una instancia mediadora que conecte estos ámbitos inconmensurables, tratándose en este caso del carácter apofántico del verbo. Esta labor de mediación es la que disuelve la muerte y la que introduce la vida en el espacio que dejan los dos primeros segmentos recíprocos.

Palabras clave: Esencia, círculo, hombre, naturaleza, mediación
\end{abstract}

\begin{abstract}
We'll try to analyze the concept of death with special emphasis on the Stuttgarter Privatvorlesungen to establish that the philosopher uses this concept with different meanings, which leads him to encompass the perspective of essence and of the entity as areas of death always that they are considered isolated. There must be between these two parameters a mediating instance that connects these immeasurable scopes, which is in this case the apophantic character of verb. This mediation is what dissolves death and what introduces life into the space left by the first two reciprocal segments.
\end{abstract}

Keywords: Essence, circle, man, nature, mediation.

Recibido: 05/10/2019. Aceptado: 05/12/2020.

* Universidad Complutense de Madrid. Colaborador Honorífico. Líneas de investigación: Idealismo alemán, Metafísica, Filosofía del Derecho. Otros trabajos en "La cuestión doble del fundamento y el principio de identidad en las Lecciones de Erlangen de F.W.J. Schelling “, en Pensamiento. Revista de Investigación e Información filosófica, Vol. 75, n 284, 2019, Universidad Pontificia Comillas, Madrid, pp. 765-786. ISSN. 0031-4749. "Schelling and the different forms of State from his New deduction of Natural Law", en Diacrítica: revista do Centro de estudos humanísticos. Cosmopolitan Challenges. 500 Anos de Utopia. Homenagem a René Girard, Vol. 30, nº 2, Húmus, 2016. ISSN. 0807-8967. Contacto: miguelramirezcordon@ gmail.com 


\section{Introducción}

Hans Urs von Balthasar, de un modo fundacional, proponía en Apokalypse der deutschen Seele (1937-39) que la muerte bajo el Idealismo alemán, jugaba el papel de mediadora entre la finitud del hombre y su naturaleza ideal. Son exiguos, en cambio, los estudios específicos sobre Schelling en abordar el elemento de la muerte y que hayan tenido un claro rendimiento dentro de los resultados estrictamente filosóficos. La muerte, en estos estudios, únicamente aflora como un concepto asociado generalmente a términos de finitud. Así ocurre en el libro de Wieland Schellings Lehre von der Zeit de 1956. Frente a este tipo de lectura se opone la de la visión de la muerte como concepto límite desde el que el ser es comprendido en la totalidad de sus determinaciones, como ocurre en la lectura de Schulz $(1955,293)$.

Es frecuente en la bibliografía especializada sobre Schelling encontrar interpretaciones irreconciliables. La apuesta que aquí quiere hacerse será fiel al planteamiento dialéctico de la filosofía idealista. Ello quiere decir que Schelling no renuncia a ambos supuestos: el de la muerte como lo propio de la finitud, pero también como lo propio de la infinitud. No hay ningún destino de finalidad concreto para este concepto, sino que un terreno y otro se conjugan como condición para alcanzar su significación dentro de su campo concreto. La muerte no es únicamente el término final de la reductio ad essentiam como propone Österreich (2002, 48), sino que esta esencia, que se ha nutrido de los términos de lo limitado para constituirse en tanto que Absoluto, se disuelve necesariamente, bajo la capacidad apofántica del hombre, para reducirse ella misma también en la finitud.

En este sentido, la esencia, en Schelling, es una Unidad que contiene, como en el espejo (S.W., IV, 317), elementos opuestos y mutuamente desdoblados. La esencia depende del hallazgo de la especificidad de la cosa. Si se dice que la cosa ha sido reducida a su esencia ello puede declararse únicamente de un modo nominal, pues la especificidad de la cosa siempre está ligada a su materia (S.W., V, 496-497)1. De este modo, concluir la esencia de la cosa por la ilustración por parte del sujeto no supone puerto de llegada porque la esencia no se da nunca en un estado de limpidez, y porque el sujeto también depende de lo objetivo para establecer sus afirmaciones $(S . W ., \text { IV, 314-315) })^{2}$. El problema de esta situación no afecta ya tanto a la cosa en sí. De ahí el cambio de la pregunta en Schelling que deja de preocuparse tanto por la cosa como por el sujeto del siguiente modo: «¿Cómo es posible esta separación en Dios?» $(S . W .$, IV , 317) , si es que, como parece querer plantear la filosofía, Dios es lo incondicionado ${ }^{4}$. Esta incondicionalidad, al final de Stuttgart, aparece asociada

1 Se ha cotejado con Schelling, F.W.J.; "Exposé de la philosophie rationnelle pure", en Introduction à la philosophie de la Mythologie, trad. GDR Schellingiana, Gallimard, Paris, 1998, pp. 297-298, donde, siguiendo a Aristóteles, confirma que la esencia consiste en el ser efectivo. Cf. Aristóteles; Metafísica, 1044a.

2 Es interesante rescatar la opinión de Hamilton Grant, I.; “«Philosophy become genetic»: The Physics of the World Soul”, en The new Schelling, Norman, J. y Welchman, A. (eds.), Continuum, 2004, London, p. 133, para quien Schelling, en su Naturphilosophie, está retomando las tesis de Kielmeyer según la cual la organización de las cosas se produce gracias a elementos heterogéneos, lo cual fuerza a una naturaleza constructiva o genética del entendimiento.

3 Más adelante, en $S$. W. IV, p. 322 certifica que este sujeto, visto en tanto que fuente de la predicación, cabe verlo como el concepto de Dios.

4 El inicio de la filosofía tiene su punto ante el asombro de la inexplicabilidad de lo incondicionado. Pero en este ámbito no son posibles las respuestas. Dado que el tránsito de lo infinito a lo finito es impracticable, debe ensa- 
a la muerte como el momento supremo de la fase ideal del Espíritu. Quizá por eso, y aunque en el Freiheitsschrift también irrumpe el concepto de muerte de un modo destacado, tomamos las Stuttgarter Privatvorlesungen como lugar donde la muerte surge de un modo más tematizado para explicar el periplo dialéctico de lo Absoluto para su autoconstitución. Así, para llegar a este episodio final también se adentrará en el concepto de lo mortal como ámbito de afectación de la materia. Hay al menos, por tanto, dos acepciones del concepto de muerte que resultan contradictorias entre sí y que deben de ser situadas en sus respectivos términos, pues la muerte en su significación material sirve de puente para llegar a la muerte en su sentido espiritual, y este último modo servirá, por contraposición, para especificar el terreno de significación de la finitud.

\section{Tesis: la muerte como dominio de lo material}

La esencia comporta una naturaleza doble porque siempre queda referida al objeto. En cambio, si dijésemos que la esencia es lo que aguarda a derramarse sobre cada objeto cabría postular que la esencia es lo ligado irresistiblemente a la cosa. La esencia entonces debe esperar el momento en el que allegarse desde una realidad originaria hasta producir las entidades particulares $\left(S . W .\right.$, IV, 25-26) ${ }^{5}$. Pero entonces lo incondicionado estará tan determinado como lo están las cosas mismas por la fuente desde la que cabe predicar el origen de la finitud, y ambos parámetros estarán mutuamente condicionados pudiendo ir desde un polo a otro de la determinación (S.W., III, 132).

Lo conocido gracias al fundamento que permite conocer deviene algo inmóvil, estático. Una vez que queda puesto el fundamento de toda predicación queda establecido el parámetro de la predicación misma, y las coordenadas de lo fijado por la apófansis queda sin posibilidad de desplazarse de los quicios que impone el orden de la fundamentación. El orden de lo dicho queda entonces embutido en el orden de relaciones que determina el

yarse el itinerario inverso. El punto de vista comienza a tornar, desde este punto, hacia las consideraciones que pueden hacerse desde el yo trascendental, como plantea Esposito, J. L.; Schelling's Idealism and Philosophy of Nature, Associated University Presses, 1977, New Jersey, p. 43. En su lúcido examen de las Cartas, Gérard, G.; 'L'absolu et la question de l'homme dans les Lettres philosophiques sur le dogmatisme et le criticisme de Schelling”, en Études d'anthropologie philosophique, Florival, G. (Ed.), Éditions de l'institut supérieur de philosophie, 1984, Louvain-la-Neuve, pp. 127-132 plantea la finitud como comienzo imprescindible del Yo particular para poder llegar a la afirmación de la tesis, la incondicionalidad del Absoluto, a la que tiende la filosofía. Sobre una afirmación como esta Cardona Suárez, L. F. en "La metafísica schellingniana del Yo absoluto como una «ética a lo Spinoza»", en Universitas Philosophica, 57, 2011, Bogotá, p. 90, puede decir que el Absoluto encuentra su legitimidad en la conciencia humana, siendo este el argumento fundamental que supondrá el respaldo a la filosofía práctica en las Cartas.

5 Schelling se distancia explícitamente de las posturas emanantistas que se iniciaría con el neoplatonismo de Plotino. En concreto, a quien tiene Schelling en mente aquí es a Eschenmayer, para quien el Absoluto no es cognoscible por sí mismo más que por la creencia en él. La vía de conocimiento del Absoluto entonces es la fe, pero no hay ninguna demostración más allá de ésta. Si el Absoluto, en cambio, aspira a ser la naturaleza racional con la que decir las cosas, la razón misma viene a ser la materia misma de conocimiento interno del Absoluto, que en el caso del hombre se hace explícito por medio de algo indemostrable como la fe. Por ello Dios o el Absoluto es siempre lo que queda del otro lado de la predicación, o es el presupuesto, el tras-fondo de la predicación. Para esta cuestión Hatem, J.; "Dieu comme personne dans la philosophie de Schelling: la Réponse a Eschenmayer constamment rapportée au Traité sur la libertê", en Annales de Philosophie, Vol. 7, 1986, Beyrouth, p. 28 ; p. 34. 
fundamento del «Todo Único», y así, lo dicho no puede desplegarse por sí mismo, sino que su margen de maniobra queda fiscalizado por el límite de oposición de la fundamentación. Del ser que queda recluido de esta manera en el orden de esta fundamentación que vendría a denominarse Dios, o lo incondicionado, podemos decir que sería un ser inactivo, un ser, en definitiva, muerto, que no puede imponerse al fundamento desde el que se insuflan sus notas $^{6}$. Pero si esta línea de predicación deja patente el orden de dependencia de los dos extremos del segmento, entonces el fundamento, Dios, resulta un ente (das Seyende) que está por lo creado y cuya presencia depende de la pervivencia del objeto. Dios queda limitado, pues, por aquello que ha creado, y lo creado deja patente el espectro de posibilidades bajo las que cabe poder expresar el carácter de un Dios que ha creado las cosas que ha creado ${ }^{7}$.

Este modelo impone un dualismo que fuerza a una relación de términos opuestos pero que, por medio de una relación binaria, fija un vínculo que, de disolverse, pondría en riesgo la pervivencia de esta realidad coaligada. En geometría el símil es el del fragmento de una línea $\mathrm{AB}$ cuya fórmula fija una dependencia que liquida el libre desenvolvimiento de ambos puntos, que quedan siempre referidos mutuamente como sigue: $\mathrm{AB} \cap \mathrm{BA}=\mathrm{AB}$. No son términos antitéticos, sino que engloban una misma realidad, aunque no cabe señalar un punto como el otro. Cuando A está siendo A no está siendo B. Ahora, dado que forman parte de la misma línea, cabe invertir el orden de referencia y, expresando adecuadamente el sentido de la trasfusión de sentido, llegar a expresar A como conteniendo lo mismo que B, y con todo A sigue siendo A y B sigue siendo B. B es lo limitado por su opuesto, A. Pero este esquema dual también permite decir que A depende de la limitación que le impone B y sin la cual sería un mero punto en el espacio. Bajo este supuesto entonces sí cabe decir que A está siendo a la vez B, y que B está siendo, en un sentido determinado, A.

Para poder pensar lo incondicionado es necesario salir de este dualismo. El problema del esquema binario es que, dado que A y B pueden devenir idénticos por medio de su referencia mutua, su contenido queda sin ser alcanzando. Póngase así la instancia desde la que se dibujan las posiciones de A y B respectivamente. Esto añade un tercer término sobre los dos primeros desde los que se postula la condición en la que A y B se expresan y la manera en que se relacionan como tales. Si añadimos un primer término previo, A y B descienden en la escala de referencia y devienen $\mathrm{B}$ y $\mathrm{C}$ al ser una parte del juicio que emite una figura que se ha antepuesto y que denominamos A. Este esquema sería entonces: $\frac{A}{B=c}(S . W .$, IV, 314). La aparición de este tercer elemento abre la posibilidad de la especificación de la referencia que se realice. Así, cabe expresar que, respecto a la forma, difiere la posición de cada una de estas figuras. Pero si el punto de vista adoptado afecta al contenido, la referencia afecta a la esencia y los tres elementos son idénticos entre sí. Cuando se formula la oración que expresa B como B, en la

6 Se trata aquí de la condición "pronominal" o "protodinámica" que expresa Hogrebe, W. en Prädikation und Genesis. Metaphysik als Fundamentalheuristik im Ausgang von Schellings »Die Weltalter«, Suhrkamp, 1989, Frankfurt am Main, pp. 84-91 como anterior a lo "predicativo" donde el fundamento es exclusivamente nominal y no se han encontrado las positividades con las que casar el concepto.

7 Un representante paradigmático de esta postura puede verse en Leibniz. El procedimiento para el conocimiento de la naturaleza de Dios se da de un modo negativo por medio del conocimiento de los efectos que nacen de la causa por antonomasia que es Dios. El procedimiento racional de Leibniz consiste en atribuir la igualdad de los polos de los predicables, y así, si la causa final ínsita en la materia es perfecta se debe a la perfección de la primera causa. Véase Leibniz; Discurso de metafísica, Porrúa, 2003, México D.F., § 19. 
sentencia A es el presupuesto de B. Pero aquí desembocamos en la paradoja que supone expresar las cosas conforme a su esencia, dado que A y B están equiparados porque lo que queda dicho de $\mathrm{B}$ es su esencia, y la perspectiva que fija la esencia es la posición de A que imprime en $\mathrm{B}$ su decir algo de $\mathrm{B}$, de donde $\mathrm{B}$, como $\mathrm{C}$, dependen de $\mathrm{A}$, y A no puede decir nada si no se le oponen B y C, disolviéndose también en este punto, aparentemente, la incondicionalidad que se buscaba. Esta primera perspectiva pone el peso en la instancia desde la que se predica. Desde aquí B no es sino el orden de las notas que impone A, de donde B, al depender de A, queda limitado igual que lo estaban los dos puntos de la recta $\mathrm{AB}$. Aquí $\mathrm{B}$ es la referencia a la esencia que nace de $A$, de donde $B$ nace, de algún modo, de $A$, por lo que B es $A(A=A)$. Ahora bien, cuando A se deshace de su cualidad de ser fuente de la predicación para hacer aflorar meramente las condiciones de $\mathrm{B}$, entonces $\mathrm{B}$ queda realzado como objeto autónomo de la predicación, y desasido de sí, A permite hacer resurgir a $\mathrm{B}(\mathrm{A}=\mathrm{B})(S . W ., \mathrm{IV}, 317)$.

Este orden jerárquico presenta un corte insalvable según el cual, desde el punto de vista de la existencia, el ente (das Seyende) se coloca como natura prius, y frente a la imposición tozuda de la materia a los sentidos, lo ideal se pone como posterius. Pero dentro de la relación que existe entre estos dos modos de mentar lo real, lo ideal es aquello que decreta el orden del ente, lo ideal es «lo superior» $(S . W .$, IV , 319). La clave para establecer la primacía de la idea frente a lo múltiple es que la conexión que se da entre las cosas no se impone arbitrariamente, sino por una voluntad precisa que deja más bien que lo ente adopte su comportamiento necesario y, bajo la observación de sus relaciones, el sujeto que es Dios pueda plegarse a ese aparecer de las cosas y simplemente constatar esa actuación ${ }^{8}$. Dios asiste voluntariamente al modo como se dispone su creación y entonces certifica cómo se ordenan las cosas, y donde Dios, al consentir en la libre actuación del cosmos, entonces también se hace libre a sí mismo al constituirse como sujeto por medio de la naturaleza (Leyte, 1998a, 95-98).

Podría pensarse que Dios ha optado por entregarse al devenir de las cosas porque en el fondo conociera ya el resultado de esa confrontación de fuerzas. Esta opción por parte de Dios estaría limitada por el conocimiento de saber que el mundo que se daría a resultas del acto creador sería el mejor de los posibles ${ }^{9}$. El estado de esta relación volvería a ser el mismo que teníamos con los puntos del segmento de una recta $(A B \cap B A=A B)$. En cambio, la elección de Dios es previa al despliegue de su obra, y por lo tanto anterior al tiempo (Carrasco Conde, 2008, 349). Forzados a pensar todo conforme a una línea causal existe el riesgo de pensar que Dios no puede sino elegir aquello que ya sabe que se va a producir. Pero es este producir lo que implica la posición de una cosa que nace como consecuencia de lo anterior. Volvemos al segmento de la línea aquí. Pero en cambio Dios sólo se determina por sí mismo, se autodetermina, no en el sentido que se dé a sí mismo su ser, sino que la opción por la que se decanta es por afirmarse a sí mismo o por autoafirmarse. Alcanzada esta difícil plataforma en la que Dios se concilia como lo que es, puede abandonarse al libre despliegue de su elección por sí

8 Brandner, R.; Natur und Subjektivität. Zur Verständnis des Menschseins im Anschluß an Schellings Grundlegung der Naturphilosophie, Königshausen \& Neumann, 2002, Würzburg, p. 52, donde se ve que el sujeto trascendental precisa del camino de una filosofía de la naturaleza o del objeto para constituirse.

9 Clara mención de Schelling a Leibniz en S. W. IV, p. 322. 
mismo sin temor a que irrumpan espectros distintos de lo que es Él. Esta asunción supone por tanto la aceptación de las cosas que deben salir de su carácter $\left(S . W .\right.$, IV, 321) ${ }^{10}$ :

Naturalmente ahora todo es verdad, es decir, la libertad absoluta es de nuevo absoluta necesidad. Por lo tanto, frente al argumento de la libertad absoluta no puede darse ningún otro fundamento; esta es de este modo porque es así, o sea, es auténticamente así y en esa medida necesaria.

Las cosas constituyen una estructura lógica análoga con la forma de la idea de la que proceden. La materia así parte de la idea, pero no forma parte de ella, lo cual conduce a deshacer el equívoco panteísta de decir que unas y otras sean lo mismo ${ }^{11}$. El reino de Dios, así, puede reafirmarse sobre «su trono» $(S . W .$, IV, 329) que es la naturaleza misma. Pero el hecho de que se establezca que la naturaleza es lo disímil de Dios permite pensar un marco de actuación propio de las cosas. Precisamente porque Dios se complace en reflejarse en la disparidad en lugar de solazarse en la contemplación de sí mismo, es que cabe ver en este sujeto algo vivo, algo que da un paso más allá de sí y que acomete el esfuerzo de atender al modo como se establecen las relaciones de las cosas entre sí (Vetö, 2003, 45-46). Un Dios que supiera en todo momento de sí sería un Dios que no tendría que realizar ningún esfuerzo por conocerse a sí mismo y conocer su obra, pues toda esta labor estaría ya concluida. Dios entonces sería un Dios despreocupado, inactivo, un Dios muerto. Las cosas que entran en el tiempo claudican frente a otras entidades que se le imponen, y son condicionadas de este modo. En esto consiste lo vivo, en aquello que pugna por imponerse frente al resto de entes que le sirven para afirmarse por medio de la negación de lo otro. Pero si continuamos tras la pista de un Dios incondicionado, habrá de decirse ahora, frente a la suposición de un Dios muerto como el que implicaba el esquema de la línea, que Dios es más bien vida, y lo es porque sirve de referencia lógica fundamental para calificar a lo vivo. Así, si Dios constituye la vida más perfecta, la vida divina, aquello que se le opone como lo vivo, por relación a la vida más excelsa, debe ser considerado «una no-vida» (Nichtleben) (S.W., IV, 329); esto es, algo muerto, pero una muerte relativa, en función de la vida absoluta del Ente supremo.

\section{Antítesis: la muerte en el seno de Dios}

Los entes particulares están limitados en su naturaleza, de la misma manera que el modo de referirse a ellos está, también, limitado por las propias reglas del discurso. En cambio, por medio de estas mismas reglas de la apófansis, es posible agrupar ciertas entidades físicamente inintercambiables en un conjunto de notas comunes, y seguir en una escala en la que conformar las especificidades del género del discurso del que se trate en cuestión. Hay un modo, por tanto, distinto a la mera finitud con el que referirse a la naturaleza, un modo lógico, el modo que pertenece propiamente ya a la naturaleza del sujeto, y con el que se establecen enunciados

10 «Nun ist freilich alle wahre, d. h. absolute Freiheit wieder eine absolute Nothwendigkeit. Denn von einer Handlung der absoluten Freiheit läßt sich kein weiterer Grund angeben; sie ist so, weil sie so ist, d. h. sie ist schlechthin und insofern nothwendig» [La traducción es mía].

11 Sobre el curso de un pensamiento panteísta en una posible afirmación como la que se expone en el cuerpo del artículo, S. W. IV, p. 330. 
categoriales que sirven de plataforma estable para referirse siempre del mismo modo al objeto. En la medida en que dicho juicio resulta adecuado con respecto al objeto expresado, entonces «A es divino en $\mathrm{B}$ » $(S . W ., \mathrm{IV}, 334)$. Podría decirse mejor que Dios se re-afirma en B, en la coincidencia común de que $\mathrm{B}$ debe ser visto «como la totalidad de la Naturaleza» (Idem). Este otro modo como B resurge en A es bajo la forma divina o bajo una de sus naturalezas, bajo la nomenclatura del Hijo, o bajo la potencia, dice también Schelling, de la «palabra» (Idem), con el que cada cosa particular, primero, aparece diferenciada en su particularidad y, finalmente, resulta reducida a la pauta universal del concepto o de lo espiritual ${ }^{12}$. Si de la relación entre A y B acabara imponiéndose solamente la observación de la materialidad de B, entonces A queda eliminado, queda tan sólo la presencia unívoca de B, que cabe ser catalogada entonces como «sustancia mortal sin interioridad alguna» (S.W., IV, 336). Pero si, en cambio, esta naturaleza muerta puede ponerse como lo vivo, si puede, de algún modo, ser resucitada la carne, es porque en A reside B, porque A puede dar en la cuenta de B, y ello, a su vez, porque B, la naturaleza y su opacidad inerte está radicada también en Dios. No de otro modo puede Dios adentrarse en el cuerpo de la naturaleza, sino porque tiene el modo de aproximarse a él y atravesarlo con categorías que exceden y van más allá del procedimiento meramente orgánico de la naturaleza, y expresar de ese amasijo de comportamientos por instinto una pauta universal.

$\mathrm{B}$ reside entonces en $\mathrm{A}$, como una rémora que impide el relumbramiento puro de $\mathrm{A}$, y que mantiene a A preso de B, obligado a hacer repercutir sus categorías ideales sobre la materia informe de B. Hay, pues, una unión inseparable de A y B, una «asociación», dice Schelling, que es efecto de «una unión viva» $(S . W ., \mathrm{IV}, 334)$ en cuya comparecencia mutua, A en tanto que A, y B en tanto que B, hace que tiendan el uno al otro. Su constitución misma los religa y conforma en una unión perpetua. Pero más que una unión es una pugna, donde la vida debe abrirse paso a través de la muerte, y donde la muerte sirve de plataforma desde la que la vida puede reorganizarse. La materia, en este sentido, contiene la vida, pero como posibilidad siempre a ser realizada. En el momento en el que la materia se hurta a la vida para reorganizarse y se oculta al verbo, la vida que permite hacer explícita a la corteza mortal, inexpresada de la realidad, cede nuevamente a la muerte y queda sumergida, cegada en ella (S.W., IV, 336). Recaemos, bajo esta órbita, constantemente en el dualismo que mantiene a Dios próximo a la muerte. En el dualismo es más bien la muerte lo que se impone, así como en el segmento AB se difumina la línea infinita. Sin embargo, Schelling llama de nuevo la atención: pese a esta oposición, «los dos principios no hacen más que uno» $(S . W ., \mathrm{IV}, 337)$. Pero también el producto de la unión de los dos puntos del segmento conforma la unidad indisociable del segmento. Sólo reconociendo en este fenómeno una realidad cuantitativa y tratando de acudir a la relación cualitativa que deja también el producto de su unión podremos salir de esta contradicción insalvable (S.W., IV, 337-338).

Este intento de localizar el dominio que se ejerce por sus correspondientes naturalezas, el de lo real por un lado y el de lo ideal por otro, concluía en el apartado anterior a favor del polo ideal. Pero sin subir un escalón más en esta labor deductiva, lo ideal corre el riesgo de

12 Brito, E.; La création selon Schelling, Leuven University Press, 1987, Louvain, p. 150. Esta última reducción de lo real a lo ideal es, para Christian Danz, la reducción de la materia a lo espiritual que sólo puede consagrar, en cambio, una figura particular como Cristo pero que, con su muerte, resurge como preñada de contenido espiritual. Véase Danz, Ch.; Die philosophische Christologie F. W. J. Schellings, Frommann-Holzboog, Schellingiana, Band 9, 1996, Stuttgart-Bad Cannstatt, p. 118. 
caer, nuevamente, bajo el imperio de la muerte. La única escapatoria que tiene la pretendida primacía de lo ideal para reafirmarse es escindirse de esta relación real-ideal y establecer su gobierno desde una parcela superior a la de la lucha dialéctica de estos dos principios. Este nuevo estatuto no debe corresponder a la esencia, pues el sujeto cuyo discurso quiere versar sobre la esencia depende, siempre, de las figuraciones de la materia, incurriendo así en el remolino perpetuo de la idea que quiere expresar la materia escurridiza bajo las categorías ideales. Este escalafón último se corresponde por tanto con la instancia que hace posible poder demostrar la esencia de lo real. Es, si se quiere, el axioma imprescindible del que hay que partir necesariamente para que el sujeto exprese algo como algo, pero donde no se da de momento juicio alguno, sino únicamente el manejo del postulado de que existe un lugar lógico desde el que poder acomodar el aparecer múltiple de lo real. Este pre-supuesto está, ya sí, libre de toda condición. Puede decirse, ahora, que se ha encontrado lo incondicionado (S.W., IV, 314), la forma vital de Dios. Se trata de la efusión espiritual «que contiene implícitamente todas las potencialidades» (S.W., IV, 343); es decir, que las contiene como posibilidades que dependen de la serie de los factores concatenados para poner en obra sus funciones respectivas, estableciendo un equilibrio gracias al arbitraje efectuado por la potencia espiritual suprema (S.W., IV, 343-344).

Si la instancia espiritual supone un mero lugar de conciliación lógica de lo ideal y lo real, en cuyo trenzado se produce el verdadero escenario de actividad propio de la vida en el que los factores del dualismo luchan por salir a cada tiempo a flote, entonces el polo que va más allá de los puntos en liza, y que se encarga más bien de producir o de hacer posible dicha pugna será propiamente el espacio de indiferencia, el espacio de inactividad frente a la actividad frenética que se da en el segmento $\mathrm{AB}$ de lo ideal y de lo real. Si el espacio espiritual de $\mathrm{A}^{3}$ es, así, el de la mera posibilidad lógica del decir algo de $\mathrm{A}$ y de $\mathrm{B}$, sin que haya ningún interés en que el fiel de la balanza penda de uno u otro lado, un mero espacio lógico de incondicionalidad, este lugar puede quizá, también, llamarse un espacio de muerte, como muerte hay en el tiempo eterno previo al desencadenamiento del proceso de la creación en el tiempo.

\section{Síntesis: muerte como naturaleza prístina del poder decir de Dios}

En el mero espacio lógico incondicionado, lo real no es la apertura de la materia que pueda ser ordenada por lo ideal, ni lo ideal alberga el modo como expresar los distintos límites de la materia. El ser y el ente y su interrelación perpetua no se ha desarrollado toda-

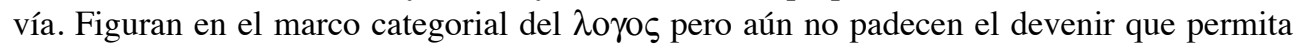
expresar los distintos modos en que puede decirse la manera en que se transforma lo ente. El ámbito espiritual en el que cada cosa puede ser expresada conforme a su esencia a partir de este espacio lógico atribuye a la composición informe de la materia su cualidad como organismo; es decir, como materia insuflada de la naturaleza conceptual que especifica su funcionamiento completo. Gracias a la comprensión del conjunto de relaciones con que comparece la cosa, asistimos a su actividad entera, y deviene así aquello que vive, aquello que tiene una serie de procesos y cambios específicos. Esta fuente de la predicación es lo que anima a la estructura orgánica de lo vivo («Deus est anima brotorum») (S.W., IV, 348). Lo que era antes lo muerto pasa ahora a ser lo viviente gracias al núcleo de categorías que parten de la unidad espiritual que aparece confirmada una vez que se comprueba la validez 
de los juicios sobre la cosa. Entonces esa fuente de validez lógica se ha confirmado, se dice que es, que existe. De este foco de validez cabe decir, si es que a partir de él confirmamos la existencia de una vida orgánica, que es «un Existente eminentemente viviente» $(S . W$. IV, 346). Pero en modo alguno puede pensarse este viviente como el carácter orgánico y viviente de la Naturaleza (Duque, 2014h, 54-55). El vínculo entre estos dos ámbitos dispares es únicamente analógico, no real. La naturaleza propiamente espiritual se juega no en ingresar en la estructura corporal de la materia, sino en trasladar la validez conceptual sobre la materia sin convertirse ella ni amenazar con tocar la materia. Se trata, pues, de un Existente esencial que propiamente no existe, que simplemente es, que está radicado en un ámbito de simple posibilidad absoluta, pero que debe aguardar hasta encontrar a un sujeto propicio con el que allegarse a las cosas y obtener su traducción precisa por parte de este sujeto, por parte del Hijo, del «Espíritu finito» (S.W., IV, 348-349). La naturaleza de este peculiar ente consiste en su propia capacidad apofántica con la que es totalmente inconmensurable ${ }^{13}$. La naturaleza de Dios está incapacitada para esta proeza. No puede moverse en ese sentido, es propiamente lo muerto que tiene la posibilidad de la vida activa pero que, por sí sólo, no puede seguir ningún curso, aunque contenga en sí todo el movimiento.

Nos encontramos, así, con dos estadios de muerte relativa, la de lo no-existente, la materia que no ha recibido aún el bautizo del nombre por parte del Espíritu, y la del Existente necesario que no puede por sí solo aprender el camino hacia la vida activa. Pese a que topamos aquí con dos conceptos de muerte equivalentes, estos no están en relación, más allá de la analogía que cabe establecer entre ambos, y con la que, en realidad, no puede entenderse el estatuto del Existente necesario, al menos no positivamente (S.W., IV, 347-348). No conforman, por tanto, los dos extremos de un segmento que haga de estas dos muertes algo intercambiable. Son más bien, si se quiere, dos segmentos que corren paralelos y que no pueden tocarse: $\mathrm{AB} \cap \mathrm{BA}=\mathrm{AB}$ y $\mathrm{AB} \cap \mathrm{BA}=\mathrm{AB}$.

Dios no toca la materia, y en ese sentido no depende de ella, así como, en la línea, A depende de B para confirmar su naturaleza y a la inversa. Y es gracias a esta realidad independiente de lo puramente lógico, y a su independencia de lo corpóreo que Dios como lo Existente necesario es auténticamente lo incondicionado, es de veras, ahora, «una libertad absoluta» (S.W., IV, 349). El ser de Dios se juega en que no pueda traspasar la franja de su línea $(\mathrm{AB})$ y alcanzar las fronteras de la línea de su creación $\left(\mathrm{AB}^{\prime}\right)^{14}$. Es por ello por lo que Dios precisa una mediación donde Dios se manifieste como tal en su creación, sin ser propiamente su naturaleza la que irrumpa en el seno de la finitud misma

13 La propia naturaleza del hombre se inscribe en la finitud, de donde adquiere poder decir las cosas de la finitud de un modo propio. Esta finitud expresa, precisamente, su naturaleza mortal, pero esa frustración ante su destino es la posibilidad para acercarse a la Vida por antonomasia de la esencia gracias al ejercicio de su libertad, como insiste Wieland, W.; Schellings Lehre von der Zeit, Carl Winter, 1956, Heidelberg, pp. 46-47.

14 La diferencia con Fichte aquí es crucial, pues para el de Rammenau, a tenor de la Wissenschaftslehre de 1810, Dios no puede ser un concepto muerto, sino que es vida límpida, lo cual se juega en que no puede cambiar en su propio concepto. Si lo pusiéramos en relación con las cosas que crea cabría suponer, entonces, que tampoco las cosas mutan. Pero para Schelling la naturaleza es lo cambiante donde, en cambio, su determinibilidad depende de la actividad propia de la razón. Dios, en este sentido, es el Prius que posibilita decir algo de algo, sin poder decir por ello que Dios comparte el estatuto de las cosas que cambian. La razón así no es, como en Fichte o en Hegel, lo inmanente, sino lo trascendente con respecto a lo creado. Véase Schulz, W.; Die Vollendung des deutschen Idealismus in der Spätphilosophie Schellings, W. Kohlhammer Verlag, 1955, Stuttgart, p. 66. 
del mundo. Esta mediación es lo que se ha llamado en la segunda parte de las Conferencias de Stuttgart "Verbo", y que en la tercera parte viene a denominarse como «hombre». Es la única naturaleza que alberga, pues, algo de las dos naturalezas que aspiran a ponerse en contacto, a recobrarse mutuamente: la infinitud de Dios y la finitud de su creación. El hombre, así, guarda, dice Schelling, «una doble relación, la del ser y la del no-ser»; es decir, guarda el vínculo con la fuente lógica de la predicación que es Dios, pero también lo hace con aquello intocado aún por las categorías del entendimiento y que, por tanto, no-es, y por ello también Schelling afirma que el hombre pertenece al mismo tiempo al ámbito espiritual y al ámbito de lo real (S.W., IV, 350; Tilliette, 1970, 457-459). Ahora bien, si nos ocupáramos ahora en analizar la naturaleza de este tercer término, de esta mediación que parece haber surgido como única tabla de salvación que puede reconciliar a Dios con su creación, observamos que verdaderamente no es en sí mismo la fuente lógica de la predicación, pero tampoco es eminentemente el marasmo caótico de la materia "natura sua". Es, de momento, lo indeterminado, y en esa medida toca, al mismo tiempo, tanto la materia muerta que no ha recibido aún determinación alguna, como también la posibilidad de la determinación absoluta igualmente muerta porque nada ha podido determinar aún.

Si decíamos que una línea es la que constituye la naturaleza de Dios, y que otra línea es la que supone la realidad de la Naturaleza, y que ambas líneas deben permanecer intocadas, la figura encargada de poner en contacto estas dos realidades, pero evitando siempre el contacto, será aquella encargada de circunscribir las dos líneas bajo una misma figura de carácter completivo. Esta figura sería el círculo ${ }^{15}$ :

A

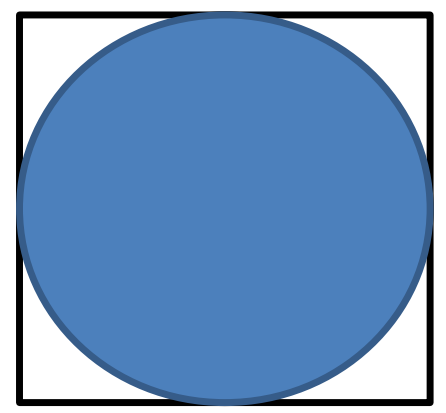

$\mathrm{A}^{\prime}$

B'

Una figura como esta, y no sino en esto estriba el concepto de hombre de Schelling, es a la que responde la conciencia en el siguiente curso que impartiera Schelling en la ciudad alemana de Erlangen. Ahí la conciencia, como distinta de la eterna libertad que se correspondería, según nuestro esquema, a la naturaleza de Dios en su limpidez, no puede

15 Sobre los problemas del círculo en la estructura de la esencia véase Duque, F.; "Schelling. La naturaleza - en Dios, o los problemas de un guion”, en Daimon. Revista de filosofía, $\mathrm{n}^{\circ}$ 40, 2007, p. 23, pero en general todo el artículo. 
residir de una manera estanca en la estancia de la «calma interioridad» (stille Innere) $(S . W ., \mathrm{V}, 33)^{16}$ de la naturaleza de Dios y debe desprenderse de esa condición ilimitada y reconocerse a sí misma siempre, constante y permanentemente en un movimiento que no es como el de Dios, eterno, sino sometido a una «necesidad ineluctable» (Idem) propia de su naturaleza. Esa necesidad es la de la conciencia humana misma que, desprendida de la unidad absoluta, transita perpetuamente desde la realidad de los conceptos puros hasta los conceptos del entendimiento que son puestos a trabajar en una labor de búsqueda de las correspondencias sucesivas que pueden ir dándose en la realidad de las cosas mundanas (Tillich, 1959, 60-62). A esta característica mudable, siempre recurrente del círculo que vuelve sobre sí mismo, pero que debe partir de nuevo desde un punto para alejarse de sí y volver a sí, lo llama Schelling «el veneno», lo llama «la enfermedad» (Cardona Suárez, 2002, 235-236), y precisamente esta naturaleza cambiante que no puede residir firmemente en conceptos claros y distintos e iguales siempre a sí mismos es para Schelling, como contrapunto a Dios, «la Muerte» (S.W., IV, 351).

\section{Conclusión: muerte como paradoja en la vía del conocimiento absoluto}

La quietud o la simplicidad de Dios, por tanto, no es la única que merece el apelativo de la muerte, sino que también la desenfrenada rueda de la conciencia recibe esta misma definición. En la misma página de las Conferencias de Stuttgart en la que el hombre aparece catalogado como responsable de este movimiento mortal, se adentra en la razón de este concepto que nos ayuda a comprender que se trata de una acepción distinta o ulterior de la muerte. Ocurre así que el hombre parte inicialmente de un «orden superior», del estadio prístino en el que posee los conceptos sin tener aún su parangón con la realidad. Pero, como veíamos, inmediatamente debe descender de ese escalón inmaculado y comprobar la validez de esos conceptos con las cosas finitas. Este descenso a que le obliga continuamente la constitución circular de la conciencia supone una fase retrógrada, una fase de acceso al ente que supone «la primera potencialidad» (Idem). Se trata, por tanto, de un estado potencial, ignorante e inconsciente, una fase de error, una estadía en lo «no existente» (Nichtseyenden) (S.W., IV, 351), y que supone el impulso a retornar a los conceptos eternos (Maesschalck, 1989, 248).

No supone el carácter reflexivo de la conciencia su naturaleza mortal sino, bien al contrario, únicamente el periodo preliminar en el que debe recolectar los datos del mundo. En la naturaleza en la que una serie de cosas singulares cobran autonomía frente a entidades iguales lo que prima, dice Schelling, es el Mal que imposibilita poder reunir las cosas que son iguales entre sí bajo un universal (Carrasco Conde, 2014, 250-251). La muerte a la que se refiere por tanto Schelling a la hora de catalogar al ámbito de lo ente se refiere a la imposibilidad de atribuir a cosas iguales etiquetas englobadoras que permitan distinguir unas cosas de otras, y donde por tanto reina el caos de los entes indiferenciados, y donde podemos decir que tenemos únicamente la lucha perpetua de las

16 La interioridad calma, acallada podemos decir, de la que habla Schelling en Erlangen es la de la eterna libertad (ewigen Freiheit) que es por antonomasia la de Dios en el momento de su carácter potencial y previo al de la verbalización de su Creación. 
cosas en liza entre sí, que tenemos por tanto una indefinición, una nada o, finalmente, el ámbito de la muerte inexpresiva de lo ente.

El término de la muerte en el que se concita la unidad de Dios es, no obstante, análogo al de lo óntico, y por ello Schelling se permite tildar al segmento AB de Dios, y al segmento $\mathrm{AB}^{\prime}$ de lo ente como muerte. En Dios se reúne la totalidad de las determinaciones, pero estas determinaciones carecen de objeto al que referirse. Son determinaciones sin juicio y por tanto su resultado es el de una nada que nada puede expresar y a nada puede referirse, y en este sentido la muerte afecta igualmente a un Dios cuyas categorías únicamente larvan su carácter potencial. La única solución que cabe introducir en el equívoco que impone esta doble acepción de muerte es plantear una naturaleza que conoce de las dos y puede circular de una a otra; esto es: la conciencia. Así, la exigencia que plantea Schelling para esta conciencia es precisamente tener que salir de la muerte que está del otro lado de los conceptos, y actualizar las cosas del mundo conforme a estos mismos conceptos y, abandonando esta primera potencia, ingresar en la segunda potencia; es decir, acceder plenamente al espacio de los conceptos puros y «que es la muerte» ${ }^{17}$. Ahora bien, este reingreso en la fuente de las categorías no tiene lugar de un modo virginal. El hombre ha accedido ya y se ha engolfado en el mundo de las cosas diversas y cuando accede al mundo ideal lo hace con la carga culposa de la percepción directa de las cosas donde, las cosas iguales entre sí, simplemente se oponían por una relación de contigüidad ${ }^{18}$. El movimiento pendular de ida y venida de la conciencia propicia que el mal de la naturaleza, el mal de lo real, esté mezclado con el bien de lo ideal y a la recíproca, que el ámbito de las categorías del entendimiento tenga el registro de las cosas a las que poder nombrar por el ensayo que ha llevado a cabo la conciencia a la hora de probar a decir las cosas con conceptos.

En su circuito la conciencia no deja nada atrás, sino que lo transporta consigo y lo lleva de un ámbito a otro permanentemente, tan pronto como la circunferencia vuelve a iniciar su tránsito ora hacia lo alto, ora hacia lo bajo. El hombre, insiste Schelling de este modo, no ingresa en el mundo espiritual únicamente como espíritu, sino que lleva consigo a su vez su naturaleza corporal y lo que de su paso por la realidad corpórea ha aprendido. Hacia el final de estas lecciones puede por fin decirse que (S.W., IV, 368)

17 S. W. IV, p. 366. Kasper, W.; Das Absolute in der Geschichte. Philosophie und Theologie der Geschichte in der Spätphilosophie Schellings, Matthias-Grünewald, 1965, Mainz, p. 405, donde el reingreso de las cosas a su «Essentifikation» es el camino de vuelta o de reditus al puro concepto (mero Könnens) y de acceso por tanto a la muerte. Esta muerte no es la muerte primitiva de la primera potencia que se observa en la naturaleza estanca de Dios y la naturaleza respectivamente, sino que es una muerte enriquecida por las notas conceptuales del entendimiento, y en esa medida es una negación de esa muerte primera y, por tanto, la Vida por antonomasia. Ver Rosenau, H.; "Essentifikation. Die theonome Existenz des Menschen in Schellings Spätphilosophie”, en Schellings philosophische Anthropologie, 2002, Stuttgart-Bad Cannstatt, pp. 54-55.

18 Por eso la condición beatífica está radicada únicamente en Dios y en la naturaleza, pero nunca en el hombre, al que el deseo le hace conducirse de un lado al otro de estos polos. No hay, como sí supone Hegel, la posibilidad de que el hombre sea sabio. La sabiduría es únicamente una aspiración, pero como dice Pérez-Borbujo en "Schelling y la metafísica de la voluntad", en Contrastes. Revista Internacional de Filosofía, vol. XVII, Málaga, 2012, p. 241, la voluntad no puede «renunciar a sí misma». 
La muerte no es por tanto ninguna separación absoluta del espíritu con su cuerpo, sino tan solo una separación del espíritu con el elemento contradictorio del cuerpo, por tanto, del bien con el mal, y del mal con el bien. ${ }^{19}$.

La frontera entre el segmento $\mathrm{AB}$ y $\mathrm{AB}$ ' tiene que ver en la medida en que los consideramos como trazados en dos partes distintas del plano. Pero, en cambio, si los dos segmentos quedan hermanados a la hora de encerrar una figura dada, entonces los dos segmentos quedan referidos a una misma cosa, como ocurre en el caso de un círculo circunscrito. Gracias, por tanto, a esta figura de mediación (Courtine, 1990, 245-247), cabe establecer una identidad entre lo ideal y lo real, por medio de lo cual Dios logra ser la fuente viva desde la que poder expresar de un modo efectivo la Creación, sin caer en el error de pensar que Dios pierde su incondicionalidad al tocar el mundo óntico sino al contrario, descubrirse como el amor divino, ahora sí la Vida por antonomasia, que logra contener su obra gracias a una realidad pneumática (el alma) $(S . W .$, IV, 365), la conciencia humana que tiene inscrita la facultad espiritual del Logos (Freydberg, 2008, 97-98), que es la encargada de conectar perpetuamente durante la eternidad estos territorios trascendentes entre sí20.

\section{Bibliografía}

\section{Fuentes primarias de Schelling}

Schelling, F. W. J. (1966), Schellings Werke [S. W.] (Schröter, M., Hg.), C. H. Beck/ R., München: Oldenburg.

Fuentes primarias

Aristóteles (1994), Metafísica, Madrid: Gredos.

\section{Fuentes secundarias}

Brandner, R. (2002), Natur und Subjektivität. Zur Verständnis des Menschseins im Anschlu $\beta$ an Schellings Grundlegung der Naturphilosophie, Würzburg: Königshausen \& Neumann.

Brito, E. (1987), La création selon Schelling, Louvain: Leuven University Press.

Cardona Suárez, L. F. (2002), Inversión de los principios. La relación entre libertad y mal en Schelling, Granada: Comares.

Cardona Suárez, L. F. (2011), "La metafísica schellingniana del Yo absoluto como una «ética a lo Spinoza»", Universitas Philosophica, 57, Bogotá, pp. 87-122.

19 «Der Tod ist daher keine absolute Trennung der Geistes von dem Leib, sondern nur eine Trennung von dem Geist widersprechenden Element des Leibs, also des Guten vom Bösen und des Bösen vom Guten» [La traducción es mía].

20 Schulze, W. A.; “Gott und Mensch. Zwei Studien zum deutschen Idealismus”, en Theologische Zeitschrift, 11,1955 , pp. 429-430, donde se constata que la propia naturaleza «abyecta» del hombre le obliga a tener que reconciliarse tratando de reparar la sutura existente entre la Naturaleza y su Creador. 
Carrasco Conde, A. (2008), "Decisión y elección o la afilada sección del instante. La libertad en Schelling y la influencia de Kant en el Freitheitsschrift", Daímon. Revista Internacional de Filosofía 2, pp. 347-354.

Carrasco Conde, A. (2014), "Ens alienum. El mal desde la Naturphilosophie en F.W.J. Schelling”, El vuelo del búho: estudios sobre filosofía del Idealismo, Del Luján di Sanza, S. y López, D. M. (eds.), Buenos Aires: Prometeo, pp. 241-266.

Courtine, J.-F. (1990), Extase de la raison. Essais sur Schelling, Paris: Galilée.

Danz, Ch. (1996), Die philosophische Christologie F. W. J. Schellings, Schellingiana, Band 9, Stuttgart-Bad Cannstatt: Frommann-Holzboog.

Duque, F. (2007), "Schelling. La naturaleza - en Dios, o los problemas de un guion", Daimon. Revista de filosofía, $\mathrm{n}^{\circ}$ 40, pp. 7-27.

Duque, F. (2014) "Schelling: filosofía de la Revelación como dialéctica de la historia", Contrastes. Revista internacional de Filosofía, Suplemento 19, pp. 27-59.

Esposito, J. L. (1977), Schelling's Idealism and Philosophy of Nature, New Jersey: Associated University Presses.

Freydberg, B. (2008), Schelling's Dialogical Freedom Essay, New York: SUNY.

Gérard, G. (1984), "L'absolu et la question de l'homme dans les Lettres philosophiques sur le dogmatisme et le criticisme de Schelling", Études d'anthropologie philosophique, Florival, G. (Ed.), Louvain-la-Neuve: Éditions de l'institut supérieur de philosophie, pp. 116-155.

Hamilton Grant, I. (2004), “«Philosophy become genetic»: The Physics of the World Soul”, The new Schelling, Norman, J. y Welchman, A. (eds.), London: Continuum pp. 128-150.

Hatem, J. (1986), "Dieu comme personne dans la philosophie de Schelling: la Réponse a Eschenmayer constamment rapportée au Traité sur la liberté", Annales de Philosophie, Vol. 7, Beyrouth, pp. 27-57.

Hogrebe, W. (1989), Prädikation und Genesis. Metaphysik als Fundamentalheuristik im Ausgang von Schellings »Die Weltalter«, Frankfurt am Main: Suhrkamp.

Kasper, W. (1965), Das Absolute in der Geschichte. Philosophie und Theologie der Geschichte in der Spätphilosophie Schellings, Mainz: Matthias-Grünewald.

Leyte, A. (1998), "Schelling: una biología teológica", Thémata: revista de filosofía, Núm. 20, pp. 89-106.

Maesschalck, M. (1989), Philosophie et révélation dans l'itinéraire de Schelling, Leuven: Éditions de l'institut supérieur de philosophie Louvain-la-neuve, Éditions Peeters.

Österreich, P. L. (2002), "Die Freiheit, der Irrtum, der Tod und die Geisterwelt. Schellings anthropologischer Übergang in die Metaphysic", Schellings philosophische Anthropologie, Stuttgart-Bad Cannstatt: Frommann-holzboog, pp. 23-50.

Pérez-Borbujo, F. (2012) "Schelling y la metafísica de la voluntad", Contrastes. Revista Internacional de Filosofía, vol. XVII, Málaga, pp. 229-246.

Popa, D. (2010), "La difficile communauté des libertés. Droit et résistance chez Fichte et Schelling en 1796-1797", Les carnets du centre de philosophie du droit, $\mathrm{n}^{\circ} 149$ : Université Catholique de Louvain, Louvain-la-Neuve, pp. 3-25.

Rosenau, H. (2002), "Essentifikation. Die theonome Existenz des Menschen in Schellings Spätphilosophie", Schellings philosophische Anthropologie, Stuttgart-Bad Cannstatt: Frommann-holzboog, pp. 51-74. 
Schulz, W. (1955), Die Vollendung des deutschen Idealismus in der Spätphilosophie Schellings, Stuttgart: W. Kohlhammer Verlag.

Schulze, W. A. (1955), “Gott und Mensch. Zwei Studien zum deutschen Idealismus”, Theologische Zeitschrift, 11, pp. 426-436.

Tilliette, X. (1970), Schelling. Une Philosophie en Devenir. La Dernière Philosophie 18211854, Vol. II, Paris: Vrin.

Tillich, P. (1959), Mystik und Schuldbewußtsein in Schellings philosophischer Entwicklung, Frühe Hauptwerke. Gesammelte Werke, Band I, Stuttgart: Evangelisches Verlagswerk.

Vetö, M. (2003), "Philosophie de l'existence et théisme scientifique. Perspectives du système schellingien", Annales de Philosophie, Vol. 24, Beyrouth, pp. 41-58.

Wieland, W. (1956), Schellings Lehre von der Zeit, Heidelberg: Carl Winter. 